\title{
Personalização e Implantação de Procedimentos de Gestão da Qualidade dentro de um Programa de Melhoria
}

\author{
Daniela Alvim Seabra dos Santos Bruno Santos Pimentel Fabiana Trindade Machado \\ Gisele Silva Cardoso Wilson de Pádua Paula Filho \\ Synergia - Universidade Federal de Minas Gerais \\ Av. Antonio Carlos 6627 - Campus Pampulha - CEP 31270-010 - Belo Horizonte - MG \\ \{daniela, brunosp, fabi, gisele, wilson\}@ dcc.ufmg.br
}

Resumo

Neste artigo descrevemos uma experiência com um programa de melhoria de organizações técnicas, nos aspectos de processos, pessoas e tecnologia, focalizando problemas da Gestão da Qualidade (Garantia da qualidade, Gestão de configurações e Gestão de revisões). Constatou-se que o diagnóstico e as recomendações do programa foram fundamentais para definir quais procedimentos da organização precisavam ser alterados, quais requeriam apenas um detalhamento e quais necessitavam de reestruturação profunda. Sem o programa, a Gestão da Qualidade continuaria sendo relegada a um segundo plano dentro da organização, não produtiva e realizada apenas para o cumprimento formal do processo oficial.

Palavras-chave: Programa de melhoria, Praxis, Gestão da qualidade, Garantia da qualidade, Gestão de revisões, Gestão de configurações.

Abstract
In this paper we describe our experience with an organizational improvement program, focusing aspects of Quality Management (Quality assurance, Reviews management and Configuration management). The diagnosis and the recommendations of the program were fundamental in defining the organizational procedures that needed change, refinement or restructuring. Without this program, Quality Management would still be playing a secondary, unproductive role inside the organization, being done just for the sake of the official process.

Key words: Improvement program, Praxis, Quality management, Quality assurance, Reviews management, Configuration management.

\section{Introdução}

A simples existência e implantação de um processo oficial e definido não são suficientes para garantir o sucesso de uma organização desenvolvedora de software. O processo real, praticado pelos engenheiros de software, deve estar alinhado com o processo oficial documentado, que, por sua vez, deve ser adequado aos objetivos e características da organização. Caso contrário, o processo oficial não é espelhado pelo real, e torna-se apenas mais um entrave burocrático.

Essa melhoria do processo real deve ser realizada adotando-se um programa de melhoria da organização em processos, pessoas e tecnologia, que contemple as necessidades da organização. Para fazê-lo de forma sistemática, verificável, controlável e repetível, é preciso que esse programa também seja conduzido de acordo com um processo bem definido e documentado. Para esse fim, a organização dos autores desenvolveu um processo para programas de melhoria.

Este artigo está organizado da seguinte forma. Na Seção 2, descrevem-se os trabalhos relacionados. A Seção 3 descreve as características relevantes do processo de desenvolvimento de software adotado na organização. Na Seção 4 detalha-se o programa de melhoria que foi utilizado neste relato. As Seções 5, 6 e 7 descrevem os resultados do programa de melhoria nas diversas subáreas da Gestão da Qualidade para a organização. Finalmente, a Seção 8 apresenta conclusões e propostas para trabalhos futuros. 


\section{Trabalhos Relacionados}

Um estudo sobre a implantação de programas de qualidade é descrito em [12]. A autora apresenta uma metodologia para avaliação e melhoria da gestão da qualidade em empresas de qualquer natureza ou porte, utilizando os critérios de excelência definidos no Prêmio Nacional de Qualidade [11]. O estudo mostra que a maioria dos problemas na manutenção de programas de qualidade se deve à inadequação de aspectos da infraestrutura da organização, o que foi constatado também neste trabalho. Em [1] é descrita uma experiência de aplicação do modelo IDEAL [8], cujo principal objetivo foi a obtenção de certificados de sistemas de garantia da qualidade. De maneira semelhante ao presente trabalho, o resultado da aplicação do modelo foi a definição de um processo padrão para a organização.

Neste trabalho utilizou-se o ProMOTe [13], um processo de melhoria de organizações técnicas, baseado no modelo IDEAL. Este processo é genérico o suficiente para ser aplicado tanto na implantação de modelos de maturidade como o CMMI (Capability Maturity Model Integration) [3], quanto em programas de melhoria de tecnologia e capacitação de pessoas.

\section{O Processo Praxis e a Gestão da Qualidade}

A organização dos autores utiliza o processo Praxis de desenvolvimento de software. Esse processo está direcionado para o desenvolvimento de aplicativos gráficos interativos, baseados na tecnologia orientada a objetos [9]. Como o Processo Unificado [6], o Praxis propõe um ciclo de vida formado por fases e fluxos. As fases são seqüenciais no tempo e constituem divisões maiores dos projetos. São definidas no Praxis as fases: Concepção, Elaboração, Construção e Transição.

Cada fase é uma sequiência ordenada de uma ou mais iterações; a iteração é uma unidade de gestão, e o final de cada uma delas deve representar um estado estável e bem definido do projeto. Em cada iteração podem ser executadas atividades de diversos fluxos de trabalho (chamados abreviadamente de fluxos), e é produzido um conjunto definido de artefatos (documentos, modelos e relatórios). A divisão por fluxos é orientada por disciplinas da Engenharia de Software, e cada fluxo constitui um conjunto parcialmente ordenado de atividades. Fluxos de trabalho podem ser divididos em subfluxos.

Este trabalho aborda o fluxo de Gestão da Qualidade, que visa a verificação e a garantia da qualidade dos produtos e processos de software. Focalizam-se aqui três de seus subfluxo: Garantia da Qualidade, Gestão de Revisões e Gestão de Configurações. O quarto subfluxo, Gestão da Manutenção, não foi abordado no ciclo relatado do programa de melhoria.

A Garantia da Qualidade fixa um nível de confiança na qualidade de um produto. No planejamento da qualidade especificam-se as tarefas de garantia da qualidade a serem feitas em cada iteração. Na auditoria da qualidade, que acontece ao fim da iteração, os artefatos de um projeto, que compõem a linha de base dessa iteração, devem ser verificados, analisando-se a coerência e a rastreabilidade entre os artefatos, além dos relatórios de revisões e testes. Cada linha de base aprovada representa uma referência confiável e oficial.

A Gestão de Revisões planeja, convoca e avalia revisões, principalmente revisões técnicas e inspeções, como definidas em [5]. O planejamento consiste na especificação das revisões para cada iteração. A realização envolve a convocação das respectivas reuniões, a distribuição do material a ser avaliado, a escolha dos revisores e do líder da revisão, a preparação da infra-estrutura física para a reunião, o arquivamento de relatórios de revisão e a coleta de métricas. No pós-processamento é efetuada a análise dos relatórios de revisão.

A Gestão de Configurações identifica artefatos e gerencia alterações. A gestão de linhas de base compreende a criação, verificação, alteração e extração de artefatos em uma linha de 
base. A gestão de bibliotecas de configurações compreende a verificação, backup, análise e auditoria das coleções de linhas de base, que formam as bibliotecas de gestão de configurações.

\section{O Programa de Melhoria de Processos}

O ProMOTe (Processo de Melhoria de Organizações Técnicas) é um processo destinado à implantação de melhorias relativas a processos, tecnologias e capacitação de pessoas, em organizações técnicas. Cada instanciação do ProMOTe representa um programa de capacitação específico, que pode focalizar a melhoria de processos ou tecnologias, a capacitação de pessoas, ou qualquer combinação desses fatores.

A arquitetura do ProMOTe tem como referência básica o Modelo IDEAL. Entretanto, o ProMOTe é um processo concreto e executável, com atividades, artefatos e critérios de controle bem definidos. O ProMOTe tem um ciclo de vida composto por cinco fases - Início, Diagnóstico, Estabelecimento, Ação e Lições - denominadas conforme traduções aproximadas das fases correspondentes do modelo IDEAL. Cada fase é composta por macroatividades. As macro-atividades sugeridas pelo IDEAL são contempladas, e outras macroatividades são introduzidas para melhor concretizar o processo. A duração do ciclo de um programa pode variar de organização para organização, mas ciclos muito longos não são recomendáveis, pois os benefícios do programa de melhoria demorarão mais tempo até serem obtidos, dificultando-se a manutenção do patrocínio e do envolvimento dos participantes.

$\mathrm{Na}$ organização dos autores, o principal objetivo do programa era aumentar a eficiência no cumprimento dos compromissos com os clientes, quanto a prazos, custos e qualidade. Pretendia-se também melhorar os mecanismos de acompanhamento de projetos, e eliminar a sobrecarga da equipe. Na fase de Diagnóstico foram identificados os pontos fortes e fracos de diversas áreas da organização. Para cada área, foi descrita a situação desejada. A partir desses resultados, foi possível identificar as oportunidades de melhoria, descrever uma lista de recomendações para guiar a organização até o estado desejado, e determinar a abordagem a ser utilizada para a implementação. Os resultados dessa fase foram relatados em [13].

Quanto à Gestão da Qualidade, o Diagnóstico concluiu que a organização não aplicava um nível adequado de formalismo, e não cumpria de fato os procedimentos propostos pelo Praxis. Além disso, as pessoas responsáveis pelas atividades de qualidade não tinham autonomia em relação às equipes de desenvolvimento. Optou-se então por uma abordagem que priorizasse a implantação completa e efetiva do Praxis, estabelecendo ações para viabilizar essa implantação.

Durante a fase de Estabelecimento foram formulados projetos que tinham como objetivo preparar a organização para a execução das práticas do processo oficial. Um dos projetos executados na fase de Ação foi o de criação de uma Gerência de Qualidade, independente da Gerência de Desenvolvimento, que teria acesso direto à Diretoria Executiva da organização. O principal objetivo dessa mudança foi evitar que atividades de garantia da qualidade fossem abandonadas em momentos de pressão dos projetos.

Outros projetos, relacionados a cada subfluxo da Gestão da Qualidade, também foram executados, com o objetivo de personalizar e detalhar os procedimentos propostos pelo Praxis. O detalhamento visa a fornecer à equipe um entendimento mais operacional do que deve ser feito, uma vez que o processo oficial descreve as atividades apenas em nível conceitual. A personalização, por sua vez, visa a adequar o processo às características da organização. Os projetos relacionados à área de qualidade gastaram ao todo 347 horas para serem planejados e executados (fases de Estabelecimento e Ação). Para a execução de cada projeto foram designados um gestor, um consultor e um executor. 


\section{Personalização e Implantação da Garantia da Qualidade}

O programa de melhoria detectou que nem todas as atividades de Garantia da Qualidade previstas no processo eram efetuadas de forma adequada; isso acontecia, por exemplo, com o planejamento da qualidade, as auditorias e as inspeções de desenho e testes. Diagnosticou-se também que geralmente as atividades de Garantia da Qualidade eram abandonadas em momentos de pressão dos projetos, em especial durante as fases de Construção e Transição. Além disso, mesmo quando o tempo para executar tais atividades era suficiente, não havia pessoas disponíveis, pois a equipe era toda alocada nas demais atividades do projeto.

Sobre o planejamento da qualidade, o programa diagnosticou que os gerentes de projeto resistiam em escrever o Plano da Qualidade, devido à grande redundância de informações entre esse e outros artefatos do processo. Ademais, o planejamento dos projetos não estava reservando tempo para a realização das auditorias, nem levando em consideração que uma linha de base pudesse ser reprovada. Em geral as iterações eram finalizadas por data, e não pelo cumprimento das atividades previstas de Garantia da Qualidade.

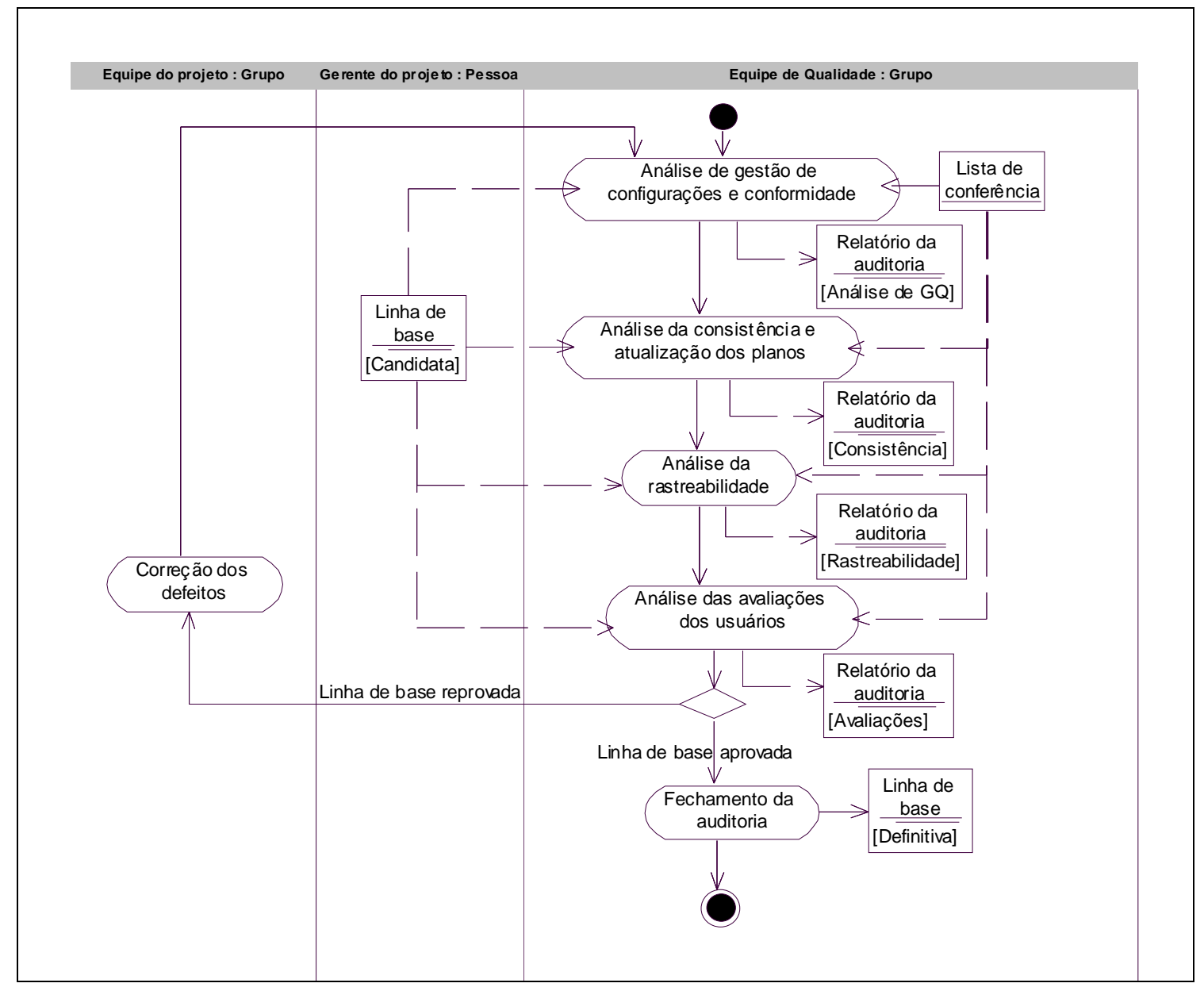

Figura 1: Diagrama de atividades da auditoria da qualidade.

Para contornar os problemas, o gabarito do Plano da Qualidade foi alterado, eliminandose as redundâncias. O plano passou a apresentar uma lista de tarefas de gestão da qualidade, única por iteração, incluindo revisões e auditorias da qualidade. Além disso, para cada tarefa 
tornou-se necessário informar a cobertura de qualidade e o período de realização da tarefa. A cobertura de qualidade define a porcentagem dos resultados produzidos (por exemplo, casos de uso) que serão submetidos às inspeções e revisões previstas no processo. A inserção deste dado torna mais visível o nível de qualidade almejado, e facilita o trabalho da Gerência de Qualidade, que irá verificar se o percentual de qualidade planejado foi realmente executado.

O plano passou a exigir também a informação das metas de qualidade para o projeto: o nível de qualidade do produto entregue ao cliente, a eficiência na correção de defeitos e a taxa de injeção destes. A inserção dessas metas passou a exigir da equipe do projeto um nível de disciplina maior com relação à qualidade dos artefatos. A Gerência de Qualidade passou a monitorar as metas de qualidade, e a compará-las com a qualidade real obtida.

Para as auditorias da qualidade, criou-se uma lista de conferência para o auditor (com cada critério classificado segundo a natureza e a gravidade), e critérios para a aprovação de uma linha de base. Esses critérios permitiram o estabelecimento de argumentos objetivos para justificar a aprovação ou reprovação de uma linha de base. A Figura 1 ilustra as atividades da auditoria da qualidade.

\section{Personalização e Implantação da Gestão de Revisões}

Para este subfluxo, o programa de melhoria detectou que, em muitos projetos, as revisões não estavam sendo planejadas, ou, se planejadas, o planejamento não era seguido, seja porque o cronograma do projeto se alterava sem replanejamento das revisões, ou seja, porque as revisões planejadas não eram previstas no orçamento do projeto.

A maioria dos defeitos encontrados em revisões e inspeções era de gravidade menor, mas isso não significava que o projeto não possuísse defeitos maiores ou críticos. Constatouse que as reuniões de revisão não eram produtivas, pois os revisores apenas relatavam os defeitos que já tinham registrado nos relatórios. Além disso, um revisor costumava encontrar os mesmos defeitos que outros participantes do grupo revisor. De forma geral, os artefatos de desenho e testes não estavam sendo inspecionados, e o pós-processamento das revisões não estava sendo feito, devido à dificuldade de extração de informações em formato textual.

A reformulação do Plano da Qualidade sanou o primeiro problema. Como já foi mencionado, o novo gabarito deste documento exige o planejamento detalhado de todas as revisões e inspeções. Introduziu-se também uma diferenciação na forma de condução das revisões técnicas e inspeções. De acordo com as sugestões de Fagan [4], quatro pessoas (um moderador, dois inspetores e o autor) constituem um bom tamanho para um grupo de revisão. Este grupo se encontra em uma reunião onde os defeitos são consolidados, as dúvidas sanadas e as duplicatas eliminadas. Constatou-se que esse formato funcionava bem para revisões técnicas de documentos para os clientes, como a Especificação de Requisitos, mas não para inspeções dos modelos de análise, desenho, testes ou implementação. Os inspetores costumavam encontrar os mesmos defeitos, e o tempo gasto durante a reunião e a consolidação dos relatórios era contraproducente.

Para inspeções de modelos foi definida, em conseqüência, uma nova forma de condução, baseada no conceito de par autor-revisor. A reunião foi abolida e o revisor, em caso de dúvida, se encontrava informalmente com o autor. Este processo, apesar de simplificar o modelo de revisões proposto por Fagan, continua seguindo seus princípios básicos: é gerado um relatório de defeitos, que devem então ser corrigidos pelo autor e ter a correção verificada pelo revisor. Por outro lado, requer menos pessoal que a programação em pares em tempo real, como é feita no XP [2].

Assim como nas auditorias da qualidade, introduziu-se o conceito da reprovação da inspeção, caso o revisor detecte um determinado número de defeitos de gravidade menor 
(erros de estilo ou de formatação) ou crítica (defeitos estruturais sérios). Na primeira situação o revisor deve interromper sua análise, pois é provável que o material ainda possua outros defeitos desse tipo, desperdiçando o tempo do revisor em reportá-los. Já a presença de um determinado número de erros críticos indica problemas estruturais sérios do material, que impedem a continuação da inspeção. Nesses casos, o material deve ser refeito pelo autor e submetido à nova inspeção. A possibilidade de reprovar uma inspeção precocemente torna a revisão mais ágil e produtiva, pois o revisor não gasta seu tempo detectando grandes quantidades de defeitos menores, ou avaliando um artefato que será reestruturado depois.

Uma outra medida tomada para melhorar as revisões foi o refinamento das inspeções sugeridas pelo Praxis, limitando-se seu escopo ao nível de caso de uso. Por exemplo, no Praxis padrão a revisão do Modelo de Análise é efetuada juntamente com a revisão técnica da Especificação dos Requisitos. Na personalização, separaram-se essas duas revisões, e criaramse inspeções dirigidas por caso de uso. Finalmente, introduziram-se inspeções por aspecto (Perspective-based Reading), que a literatura sugere serem mais eficientes que as inspeções baseadas em listas de conferência [7].

\section{Personalização e Implantação da Gestão de Configurações}

O programa de melhoria constatou que a organização não efetuava realmente a gestão de configurações. O Praxis prevê uma biblioteca de trabalho, a ser usada no dia-a-dia, com compartilhamento livre, restrito apenas pela disciplina dos programadores na execução de testes de unidade e integração, no estilo XP; e uma biblioteca oficial com as linhas de base aprovadas nas auditorias, de maior formalismo de acesso, no estilo SW-CMM [10].

Observou-se que a biblioteca oficial não estava sendo usada como referência oficial e confiável dos artefatos. Os desenvolvedores consultavam ou usavam apenas as versões da biblioteca de trabalho. O planejamento dos projetos não fazia previsão de esforço e recursos para as atividades de gestão de configurações. Finalmente, não existia uma política de restrição aos artefatos para equipes que não estavam envolvidas na sua confecção. Apesar do uso de uma ferramenta de gestão de configurações, esta servia apenas para armazenamento de informação e controle de concorrência dos arquivos.

Implantou-se uma política de permissão de acesso à biblioteca de trabalho que funciona da seguinte forma: cada artefato do projeto é armazenado numa pasta distinta, na biblioteca de trabalho, e em cada uma dessas pastas, em determinadas iterações, somente um grupo de desenvolvedores possuirá permissão de consulta, extração e inserção. Por exemplo, os desenhistas só possuem permissão de consulta nas pastas correspondentes ao Modelo de Análise até a iteração em que esse modelo deve ser finalizado. Após essa iteração, quando a biblioteca oficial já possui uma versão oficial e confiável do artefato, essa equipe deve extrair uma cópia de consulta diretamente daquela biblioteca.

O primeiro objetivo dessa política é impedir que equipes que não precisem ou não possam consultar ou modificar determinado artefato tenham meios para fazê-lo. O segundo é garantir que as equipes que precisem consultar determinado artefato sempre utilizem a versão aprovada em auditoria e confiável, presente na biblioteca oficial. A implantação dessa política reforçou a importância da auditoria da qualidade e da aprovação de uma linha de base. Uma linha de base reprovada implica em atraso no projeto, pois, enquanto ela não for aprovada nenhum artefato da iteração é armazenado na biblioteca oficial, e as equipes que usam estes artefatos como insumos ficam sem versão de consulta.

Formalizaram-se também as regras de gestão de configurações que deveriam ser observadas durante uma auditoria da qualidade. Foram definidas as regras para nomenclatura de artefatos (e partes destes), assim como a lista detalhada de artefatos (e partes destes) que 
deveriam fazer parte de cada linha de base. Finalmente, detalharam-se os procedimentos de inserção e alteração de linhas de base. $O$ último procedimento passou a exigir maior formalidade, com a entrega de uma solicitação à Gerência da Qualidade, onde detalhes sobre as alterações (data, artefato, impacto, etc...) são especificados. A Figura 2 ilustra o procedimento de criação de linha de base.

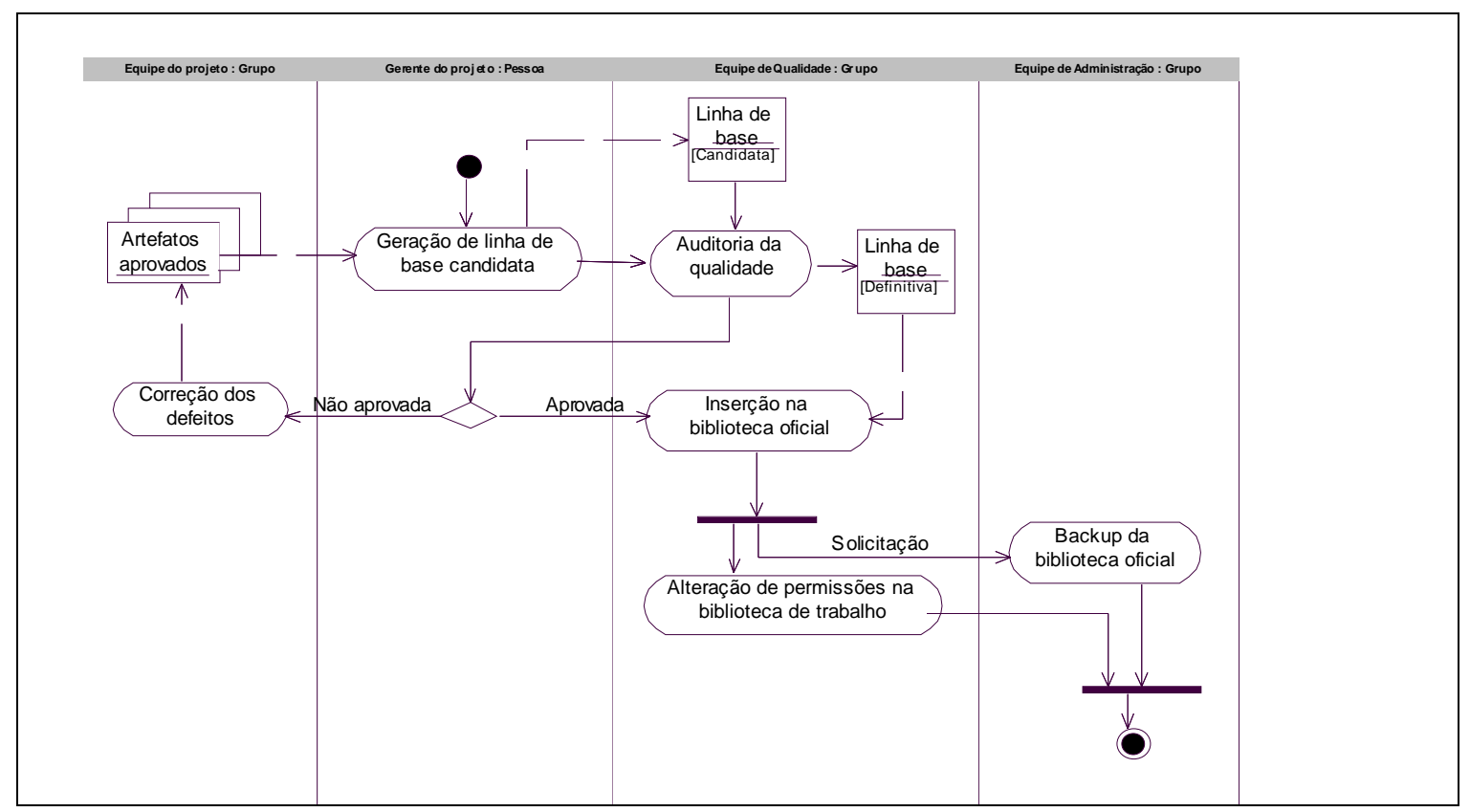

Figura 2: Diagrama de atividades da criação de uma linha de base.

\section{Conclusões e Trabalhos Futuros}

Neste trabalho relatou-se a experiência com aspectos de Gestão da Qualidade que foram personalizados e implantados na fase de Ação de um programa de melhoria da organização. Constatou-se que o diagnóstico e as recomendações do programa foram fundamentais para definir quais aspectos da organização precisavam ser alterados, quais requeriam apenas um detalhamento ou personalização, e quais necessitavam de reestruturação profunda.

$\mathrm{Na}$ Garantia da Qualidade as principais providências foram a criação de uma Gerência da Qualidade autônoma; a personalização do Plano da Qualidade, detalhando-se as tarefas da qualidade e definindo-se metas numéricas para a qualidade dos projetos; e o detalhamento dos procedimentos de auditoria da qualidade. Na Gestão de Revisões introduziu-se uma diferenciação na condução de revisões técnicas e inspeções e um refinamento das inspeções definidas pelo processo de desenvolvimento. Com relação à Gestão de Configurações, implantou-se uma política de permissão de acesso para a biblioteca de trabalho, formalizaramse as regras de gestão de configurações a serem verificadas a cada auditoria e detalharam-se os procedimentos de criação e alteração de linhas de base.

Os procedimentos de Qualidade que foram desenvolvidos de acordo com as recomendações do programa estão sendo adotados em dois projetos em andamento na organização, com tamanho aproximado, respectivamente, de 320 e 1200 pontos de função. Para cada fluxo técnico do Praxis, o gasto com atividades de revisões variou entre 10-15\% do esforço do projeto destinado às atividades do fluxo técnico em questão e essa percentagem está sendo usada no planejamento, como estimador e limitador do esforço gasto em revisões. 
Como trabalhos futuros, pretendemos efetuar uma personalização para o subfluxo de Gestão da Manutenção, da mesma forma como foi conduzida para os outros subfluxos. Além disso, planeja-se quantificar os ganhos notados com a implantação das recomendações do programa de melhoria. Será importante também estabelecer uma metodologia de análise das métricas de qualidade coletadas, de maneira a possibilitar um diagnóstico mais preciso de problemas de capacitação e do processo de desenvolvimento adotado na organização, bem como identificar ações corretivas de maneira mais ágil.

\section{Agradecimentos}

Os autores agradecem à IBM pelo apoio a este trabalho, por meio de cessão de licença acadêmica da Rational Suíte Enterprise, dentro do IBM Scholars Program.

\section{Bibliografia}

[1] E.P. Alves e R.A. Falbo. Implantando um Programa de Melhoria de Processo: Uma Experiência Prática. VIII Workshop de Qualidade de Software, XV Simpósio Brasileiro de Engenharia de Software, Rio de Janeiro, RJ, Brasil, Outubro 2001.

[2] K. Beck. Extreme Programming Explained: Embrace Change. Addison-Wesley, 1999.

[3] http://www.sei.cmu.edu/cmmi/cmmi.html

[4] M. Fagan. Design and Code Inspections to Reduce Errors in Program Development. IBM Systems Journal, 15\{3\}: 182-211, 1976.

[5] IEEE Std. 1028 - 1988. IEEE Standard for Software Reviews and Audits, in IEEE. IEEE Standards Collection - Software Engineering. IEEE, New York - NY, 1994.

[6] I. Jacobson, J. Rumbaugh e G. Booch. Unified Software Development Process. AddisonWesley, 1999.

[7] Oliver Laitenberger, Khaled El Emam e Thomas G. Harbich. An Internally Replicated Quasi-Experimental Comparison of Checklist and Perspective-Based Reading of Code Documents. IEEE Transactions on Software Engineering, 27(5), May 2001.

[8] Bob McFeeley. IDEAL: A User's Guide for Software Process Improvement. CMU/SEI96-HB-001. Software Engineering Institute, Pittsburgh - PA, February 1996.

[9] Wilson de Pádua Paula Filho, Engenharia de Software - Fundamentos, Métodos e Padrões. LTC Editora, 2a edição, 2003.

[10] Mark C. Paulk, Charles V. Weber, Bill Curtiss e Mary Beth Chrissis. The Capability Maturity Model: Guidelines for Improving the Software Process. Addison-Wesley, 1995.

[11] Prêmio Nacional da Qualidade http://www.fpnq.org.br

[12] Simone Meister Sommer. Uma Metodologia Para Avaliação e Melhoria do Processo de Gestão da Qualidade nas Empresas. Dissertação de Mestrado em Engenharia de Produção e Sistemas - Universidade Federal de Santa Catarina, 1999.

[13] Wilson de Pádua Paula Filho, Fabiana T. Machado, Fernanda P. Drumond, Márcia Mônica Nogueira e Gisele R. de M. Ferreira. Aplicação da fase de Diagnóstico de um processo para melhoria de organizações técnicas. V Simpósio Internacional de Melhoria de Processos de Software (SIMPROS’2003). Recife - PE, pp. 71-80, Out. 2003. 